\title{
Dissolution Testing Using Fiber Optics- A Regulatory Perspective
}

\author{
Vivian A. Gray \\ V. A. Gray Consulting, Inc \\ 9 Yorkridge Trail, Hockessin, DE 19707
}

\author{
Email correspondence to: vagray@rcn.com \\ This article has been reprinted with permission \\ granted from American Pharmaceutical Review, \\ Volume 6, Issue 2, Summer 2003, pages 26-30
}

\section{Introduction}

Fiber Optics for dissolution testing has been beyond the prototype stage for over five years now. It is a labor saving and efficient way to analyze and sample dissolution testing procedures. There still appears to be some resistance by companies to fully embrace this technology. One aspect of this article will be to explore a regulatory perspective on this technology. Decisions on whether to use or purchase such technology may be guided by assessing certain aspects of the equipment. A checklist will be provided to assist in this effort. Once a company has decided to use this new technology for products then how would the proper validation be performed will be explored. The method for showing equivalence to the more traditional sampling/analyzing method will be described. There will be a discussion of the utility of fiber optics in newer dosage form types and special testing.

\section{Regulatory Update}

The perception that USP or FDA may not be in favor of new technology is not an accurate assessment. We have heard that FDA and USP are not in favor of a proliferation of new equipment and this is a point easily understood. However, this does not preclude or should be intended to discourage improvements in efficiency or the science provided in new technology. There are some indications that the regulators do not discourage this fiber optic technology along with other new technologies. At USP for instance, there was a stimuli article published in Pharmacopeial Forum (PF) (1) where a new dissolution informational general chapter was put forth. In the abstract of that article, one of the purposes of the new chapter would be to give guidance to the analyst on validation and use of new technology and equipment. Under the Method Validation section in the discussion of spectrophotometric analysis, the statement is made that "Fiber optics as a determinative method, with proper validation, is an option". This article in $\mathrm{PF}$ is now the foundation of a proposal for a new general chapter in USP.

In General Chapter <851 > Spectrophotometry and Light-Scattering, there has been discussions centering on highlighting the fiber optic technology in some way. This may appear in future revisions to this chapter.

In USP General Notices (2), under the section TESTS AND ASSAYS in the Apparatus discussion, it states that "Where an instrument for physical measurement, such as a spectrophotometer, is specified in a test or assay by its distinctive name, another instrument of equivalent or greater sensitivity or accuracy may be used."The Notices go on to say, "Items capable of equal or better performance may be used if these characteristics have been validated'. In the Procedures, there is the following discussion of automated procedures:

Automated procedures employing the same basic chemistry as those assay and test procedures given in the monograph are recognized as being equivalent in their suitability for determining compliance.

Conversely, where an automated procedure is given in the monograph, manual procedures employing the same basic chemistry are recognized as being equivalent in their suitability for determining compliance. Compliance may be determined also by the use of alternative methods, chosen for advantages in accuracy, sensitivity, precision, selectivity, or adaptability to automation or computerized data reduction or in other special circumstances. Such alternative automated procedures or methods shall be validated. However, Pharmacopeial standards and procedures are interrelated; therefore, where a difference appears or in the event of dispute, only the result obtained by the procedure given in this Pharmacopeia is conclusive.

This discussion clearly paves the way for new technology as long as it is coupled with the proper validation. The FDA, in 21 CFR Part 11, the area of computer generated data is under scrutiny for accuracy and audit trails. Obviously, since there is a high degree of computerized data collection, this aspect needs to be considered when validating the fiber optic system software. Meeting the FDA regu- 


\section{Dissolution Testing Using Fiber Optics- ... continued}

lations in this area are going a long way to secure FDA approval of the new technology.

The laboratories of both FDA and USP have used fiber optic dissolution testing, a positive indication that the technology is becoming more commonplace and acceptable.

\section{Evaluating the Fiber Optics system}

There are several vendors specializing in fiber optic equipment for dissolution testing and we are lucky to have such willing and cooperative vendors when it comes to the nitty gritty of how does the equipment work in your hands or with your product.

If you are serious about purchasing the equipment, and there is management approval, it is suggested that you bring the equipment in-house for a loan. Be sure you use it on several typical formulations and have someone devote full time energy to the evaluation. Some formulators tend to use the same excipients over and over again. If this is the case, be sure those products are used for evaluation. Presented here is a checklist of major equipment parameters to evaluate when examining the various brands. This list is fairly comprehensive, but there may be aspects important to your products and operating procedures that are not covered.

1. Probes. There are different designs of probes: arch, in shaft, in resident, in resident but partially out during sampling. The probe design does appear to be stabilizing although it looks like the vendors are constantly revisiting it in response to customers.

a. Available pathlengths. Watch for availability of small pathlength cells, fiber optic probes may not be able to get down to the smaller cell pathlengths that are found with UV flow-cells.

b. Size and Shape. Is the probe container the size of a cannula or smaller, where does the cell attach? Some are arch-shape-how thin and durable is the wire?

c. Orientation of the probe. Is it put through the vessel cover or on a manifold? Is it located in the USP stated sampling zone? This sampling zone is "..midway between the surface of the Dissolution Medium and the top of the rotating basket of blade, not less than $1 \mathrm{~cm}$ from the vessel wall" (3).

d. Location of probe throughout the test. A question with conventional automated sampling that always needs to be considered is whether the sampling probes are in the dissolution medium throughout the test or just when the sample is taken. With fiber optics probes it is quite difficult to completely take the probe out, hence risk the cell drying out. Evaluate the effect of the probe on the hydrodynamics of the vessel by doing the proper validation - this will be described in the next topic of Validation.

e. Cleaning procedure. What is the best way to clean the fiber optic probe? How do you assess carryover? Do you use a water/methanol rinse? How much volume of each? How do you dry the probe? How do you store the probe?

f. Handling ease. How breakable/delicate are the cells? Ease of cable and probe manipulation, and of replacing cells should be examined.

2. Optic System Design. Some designs have each probe as a different spectrophotometer with six individual photodiode arrays (PDAs), whereas others are sequentially related to one central spectrophotometer using a channel selector that reads from each vessel serially. The optic system design has a large impact on data collection, especially timing of sampling and zero readings. The pro and cons of the spectrometric operational differences should be understood. How is the light scattering accommodated? These different designs also have an impact on the operation as seen in 3. Operation.

3. Operation.

a. Are air bubbles a problem? Always properly deaerate the medium to help alleviate this issue. Even after deaeration, before you begin the run but after the probes are in the medium, you may lightly tap the fiber optic probes to insure that no air bubbles have been caught in the cell within the probes.

b. Assess the reproducibility and precision of the standard readings, looking at each probe versus between probes. This is where the different optic design systems may become a factor.

c. How are the standards read? In a common beaker? How easy is it to read the standards? It is difficult if not impossible to bracket the standards after each timepoint as in conventional UV analysis, therefore the running of standards at the beginning and end of the run is necessary.

d. How do you mechanically set up a run, what is the compatibility with different bath models, how about sample introduction (staggered or simultaneously), can the bath operation be controlled, is there dual bath control, and how easy is the cleanup of the entire system?

e. Check for the capacity to perform and sample an infinity (fast stir) timepoint.

4. Data collection. Some systems will have baseline correction only; others have both baseline correction and second derivative. These are mathematical, com- 
puter generated ways to compensate and accommodate the turbidity and light scattering caused by excipients or undissolved drug. This aspect of the fiber optic technology is most important. Validation will be a major factor in determining the usefulness of these mechanisms.
a. Final calculation reporting- \% dissolved and/or mg dissolved?
b. Graphic printing capabilities-\% dissolved, individ- ual scans, per channel?
c. Report format-how versatile?
d. Compatibility with a LIMS system. It is very important to understand and be sure to get the company's other groups involved.
e. What is the true absorbance range versus the suggested range by the vendor?

5. Evaluate cost and cost of parts and different cell sizes. Assess the technical support. Is it worthwhile to retrofit to existing equipment?

\section{Validation}

The stage of development for your product would determine the level of validation for your fiber optic system. If you are just scouting a formulation you may only need to know the linear range to begin obtaining data. As you become closer to reportable data collection, the validation parameters that are necessary increase. Products in early development would need precision, linearity and range, and recovery (maybe just a fast stir/infinity value would be enough). But most importantly at this stage, the placebo interference or turbidity and light-scattering effects should be determined. It may be that the background correction is adequate, or if the capability exists-second derivative calculations may generate data that is accurate and useable. The background or placebo interference should be kept 2$3 \%$ or below. Some labs feel up to $5 \%$ is tolerable. If the interference can not be minimized, the traditional UV or HPLC with filtration may be necessary.

After the placebo interference has been minimized and is shown to be accurate and reportable, the last validation aspect must be considered. That is the manual versus automation (fiber optic) sampling method. This is where the hydrodynamic effect of the fiber optic probe is assessed. One method of performing this comparison could be as follows:

Perform two concurrent runs (using identical sampling time intervals, $n=6$ ) using manual and automated (fiber optic) sampling methods. This is four runs total, two manual and two using the fiber optic system. Typical acceptance criteria are that the difference in the mean value between the dissolu- tion results from the manual run versus fiber optic system run does not exceed an absolute $10 \%$ at time points with less than $85 \%$ dissolved and does not exceed $5 \%$ for those remaining time points above $85 \%$. Acceptance criteria may be product specific, and other statistical tests and limits may be used (1).

Performing a blank run after multiple runs of the highest strength may validate the cleaning procedure of the fiber optic system.

The validation for the final product as it approaches later stages/phases will include ruggedness, robustness, recovery studies, and solution and sample stability.

\section{Documentation}

At some point a validation report will be written, either as a part of an NDA or ANDA filing or as early phase documentation. It would be useful to have a special section devoted to the validation of the fiber optic system. The comparison to the manual method would be a critical part of the report. The unique aspects of the fiber optic operation, that is the cleaning of the cells, standard reading or matrix procedure, and the calculations are important. The calculations, especially the background or second derivative formulas, should be clearly explained and validated and in conformance to 21 CFR Part 11.

If there is an established method either in the USP or NDA/ANDA, a new technology such as fiber optics may be validated and used routinely. The new methodology could be filed in the next annual report, which would include the validation report.

\section{Other Products/Uses for Fiber Optic Technology}

Transdermal, powder, and intrinsic dissolution will be ideal for fiber optics, as there are no excipients to interfere with the analysis. Products where there are extrusion issues with filtration as modified parenteral products, for example, nanospheres and liposomes, may be analyzed more easily using fiber optics. Other apparatus other than USP Apparatus 1 and 2 could in the future use fiber optic probes. It would be necessary for the probe to go into a well-mixed vessel and to sample at an appropriate place.

\section{Conclusion}

There are many advantages to using a fiber optic system versus a conventional sampling and detecting method. The elimination of filtration and sample collection is a huge saving of labor and resources. The analyst should explore this new technology and feel they are within the boundaries of acceptable methodology provided the proper validation occurs. Fiber optics technology will most likely improve and more applications discovered. 


\section{Dissolution Testing Using Fiber Optics- ... continued}

A bibliography of many articles published on fiber optics is referenced to assist the analyst in becoming more knowledgeable and better trained to use the technology.

\section{Acknowledgement}

The author would like to acknowledge the contributions, particularly for the checklist, of John Ballard, DuPont Pharmaceuticals

\section{References}

1. Gray VA, Brown CK, Dressman JB, Leeson LJ. A New General Information Chapter on Dissolution, Pharm Forum 2001:27 (6): 3432-3439.

2. General Notices, USP 26, 2003: 6-7.

3. General Chapter $<711>$ Dissolution, USP 26, 2003:2156.

\section{Bibliography}

4. Earnhardt J, Nir I. Fiberoptic dissolution testing, Advances, drug quality control, Spectroscopy 2000:15(2):31-38.

5. Cho JH, Gemperline PJ, Salt A, Walker DS. UV/Visible Spectral Dissolution Monitoring by in Situ Fiber-Optic Probes, Analytical Chemistry 1995:67(17): 2858-2863.

6. Li W, Chen J, Xiang B, An D. Simultaneous on-line dissolution monitoring of multicomponent solid preparations containing vitamins $\mathrm{B} 1, \mathrm{~B} 2$, and $\mathrm{B} 6$ by fiberoptic sensor system, Analytica Chimica Acta 2000:408(2000):39-47.

7. Bynum, KC, Kraft E, Pocreva J, Ciurczak EW, Palermo P. In Situ Dissolution Testing Using A UV Fiber Optic Probe Dissolution System, Dissolution Technologies 1999:6(4): 8-10.

8. Johansson J, Cauchi M, Sungren M. Multiple fiberoptic dual-beam UV/Vis system with application to dissolution testing, Journal of Pharmaceutical and Biomedical Analysis:2002:29 (3):469-476.

9. Inman GW, Wethington E, Baughman E, Horton M, System optimization for in situ fiber-optic dissolution testing, Pharmaceutical Technology: October 2001: 92-100.

10. Bynum $\mathrm{KC}$, Kraft $\mathrm{E}$, A new technique in dissolution testing, Pharmaceutical Technology: 23 (10): 1999.

11. Dubin $\mathrm{CH}$, Presto dissolvo! Fiber otic dissolution: Magical results? Pharmaceutical Formulation and Quality: Aug./Sept 2002: 20-27.

12. Bynum K, Roinestad K, Kassis A, Pocreva J, Gehriein L,
Cheng F, Palermo P, Analytical Performance of a fiber optic probe dissolution system, Dissolution Technologies: 6 (4): 2001:13-22.

13. Schatz C, Ulmschneider M, Altermatt R, Marrer S, Evaluation of the Rainbow dynamic dissolution monitor semi-automatic fiber optic dissolution tester, Dissolution Technologies: 7 (4): 2000:8-17.

14. Schatz c, Ulmschneider $M$, Altermatt $R$, Marrer $S$, Reader's response: hollow shaft sampling with fiber optics, Dissolution Technologies: 7 (1): 2000: 20-21.

15. Allen Rl, Box KJ, Comer JEA, Peake C, Tam KY, Multiwavelength spectrophotometric determination of acid dissociation constants of ionizable drugs, Journal of Pharmaceutical and Biomedical Analysis: 17(1998):699-713.

16. Josefson $M$, Johansson $E$, Torstensson A, Optical fiber spectrometry in turbid solutions by multivariate calibration applied to tablet dissolution testing, Anal Chem: 60 (1988): 2666-2671.

17. Gemperline PJ, Cho JH, Baker B, Batchelor B, Walker $D S$, Determination of multicomponent dissolution profiles of pharmaceutical products by in situ fiberoptic UV measurements, Analytica Chimica Acta: 345 (1997): 155-159.

18. Li W, Chen J, Xiang B, An D, Simultaneous on-line dissolution monitoring of multicomponent solid preparations containing vitamins $\mathrm{B} 1, \mathrm{~B} 2$ and $\mathrm{B} 6$ by fiberoptic sensor system, Analytica Chimica Acta: 408 (2000):39-41.

19. Nir I, Johnson BD, Johansson J, Schatz C, Application of fiber-optic dissolution testing for actual products, Pharmaceutical Technology: May (2001):33-40.

20. Rogers P, Hailey PA, Johnson GA, Dight VA, Read C, Shingler A, Savage P, Roche T, Mondry J, A comprehensive and flexible approach to the automated-dissolution testing of pharmaceutical drug products incorporating direct UV-Vis fiber-optic analysis, online fluorescence analysis and off-line storage options, LRA: 12 (2000): 12-22.

21. Schatz $C$, Ulmschneider $M$, Altermatt $R$, Marrer $S$, Altorfer $\mathrm{H}$, Manual in situ fiber optic dissolution analysis in quality control, Dissolution Technologies: 7(2): 2000: 6-13.

22. Aldridge PIKE, Melvin DEW, Williams BA, Brain K, Kostek JL, Sekulic SS. A Robotic Dissolution System with On-Line Fiber Optic UV Analysis. Journal of Pharmaceutical Science:1995:84 (8) 909-914. 